\title{
The Application of Time Evolution Operators and Feynman Diagrams to Nonlinear Optics
}

\author{
AMNON YARIV, FELLOW, IEEE
}

Abstract-This paper develops a consistent formalism for describing nonlinear optical mixing and multiphoton processes of any arbitrary order. The theory uses the time-evolution operators of quantum mechanics, and the related Feynman diagrams.

\section{General BAckground}

$\mathrm{T}$ $\mathrm{HE}$ purpose of this paper is to demonstrate the use of the quantum mechanical evolution operator formalism and the closely related technique of Feynman diagrams in deriving the nonlinear constants characterizing different multiphoton nonlinear optical processes.

As an illustration we apply the method to sum and frequency generation, two-photon absorption, and various Raman processes. We start with a short review of the evolution operator formalism [1] .

The eigenfunction $\psi(t)$ of an atom subjected to an electromagnetic field can be obtained formally by solving the Schrödinger equation

$$
H \psi=i \hbar \frac{\partial \psi}{\partial t}
$$

or, equivalently, by operating on the eigenfunction at time $t_{a}$ with the evolution operator $u\left(t_{b}, t_{a}\right)$ according to

$$
\psi\left(t_{b}\right)=u\left(t_{b}, t_{a}\right) \psi\left(t_{a}\right)
$$

where $u\left(t_{b}, t_{a}\right)$ satisfies

$$
i \hbar \frac{u\left(t_{b i}, t_{a}\right)}{\partial t_{b}}=H u\left(t_{b}, t_{a}\right) \text {. }
$$

If $H$ does not depend on time, then it follows from (3) that

$$
\begin{aligned}
u\left(t_{b}, t_{a}\right) & =\exp \left[-i \frac{H}{h}\left(t_{b}-t_{a}\right)\right] \\
& =\sum_{m}|m\rangle\langle m| \exp \left[-i \omega_{m}\left(t_{b}-t_{a}\right)\right]
\end{aligned}
$$

where $\left.\omega_{m} \equiv E_{m}|\hbar| m,\right\rangle$ is the eigenfunction of $H$ with energy $E_{m}$, i.e., $H|m\rangle=h \omega_{m}|m\rangle$ and $\left.\Sigma / m\right\rangle\langle m|=I$.

In the cases of interest to us we take the Hamiltonian as

$$
H(t)=H_{0}+V(t)
$$

where $H_{0}$ is time independent and $V(t)$ represents the time dependent interaction of the atom with the optical fields.

Manuscript received July 8,1977 . This research was supported by the U.S. Army Research Office.

The author is with the California Institute of Technology, Pasadena, C.A 91109.
The solution of $u\left(t_{b}, t_{a}\right)$ when the Hamiltonian is given by (5) can be obtained by a perturbation expansion in powers of $v(t)$. The result is [1]

$$
\begin{aligned}
u\left(t_{b}, t_{a}\right)= & u^{(0)}\left(t_{b}, t_{a}\right)+u^{(1)}\left(t_{b}, t_{a}\right)+u^{(2)}\left(t_{b}, t_{a}\right) \\
& +\cdots u_{n}^{(n)}\left(t_{b}, t_{a}\right)+\cdots
\end{aligned}
$$

where

$$
\begin{aligned}
u^{(0)}\left(t_{b}, t_{a}\right)= & \exp \left[-i \frac{H_{0}}{h}\left(t_{b}-t_{a}\right)\right] \\
u^{(1)}= & \left(-\frac{i}{\hbar}\right) \int_{t_{a}}^{t_{b}} u^{(0)}\left(t_{b}, t\right) V(t) u^{(0)}\left(t, t_{a}\right) d t \\
u^{(2)}= & \left(-\frac{i}{\hbar}\right)^{2} \int_{t_{a}}^{t_{b}} \int_{t_{a}}^{t_{1}} d t_{1} d t_{2} u^{(0)}\left(t_{b}, t_{1}\right) V\left(t_{1}\right) \\
& u^{(0)}\left(t_{1}, t_{2}\right) V\left(t_{2}\right) u^{(0)}\left(t_{2}, t_{a}\right) \\
u^{(3)}= & \left(-\frac{i}{\hbar}\right)^{3} \int_{t_{a}}^{t_{b}>t_{1}>t_{t_{a}}} \int_{t_{a}}^{t_{2}} d t_{1} d t_{2} d t_{3} u^{(0)}\left(t_{b}, t_{1}\right) \\
& \cdot V\left(t_{1}\right) u^{(0)}\left(t_{1}, t_{2}\right) V\left(t_{2}\right) \\
& \times u^{(0)}\left(t_{2}, t_{3}\right) V\left(t_{3}\right) u^{(0)}\left(t_{3}, t_{a}\right)
\end{aligned}
$$

where

$$
t_{b}>t_{1}>t_{2}>t_{2}>t_{3} \cdots>t_{n} .
$$

The manner in which we are going to apply this formalism may perhaps be best illustrated by an example.

Consider the Raman scattering process in which an atom (or molecule), subjected to an optical field containing frequencies $\omega_{1}$ and $\omega_{2}$ and initially in the ground state $n$, absorbs a photon at $\omega_{1}$, emits a photon at $\omega_{2}<\omega_{1}$ while making a transition to state $k$. This process is illustrated in Fig. 1.Each scattering event results in the absorption of a photon at $\omega_{1}$ and the emission of a photon at $\omega_{2}$. We may wish to calculate the rate at which $\omega_{2}$ photons are generated per unit volume. This rate divided by the $\omega_{2}$ photon flux (photons $\cdot \mathrm{m}^{-2} \cdot \mathrm{s}^{-1}$ ) gives us the exponential gain constant of the $\omega_{2}$ field (the Raman gain).

We calculate the rate at which an atom makes the Raman transition $n \rightarrow k$ by taking the time derivative of the probability that an atom initially in the ground state $n$ will be found at time $t$ in state $k$. This probability is 


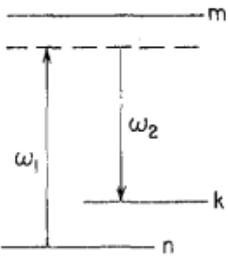

Fig. 1. Levels and frequencies involving a Raman scattering from state $n$ to $k$.

$$
\begin{aligned}
\mathscr{P}_{k}(t) & =|\langle k \mid \psi(t)\rangle|^{2} \\
& =|\langle k|u(t, 0)| n\rangle|^{2} .
\end{aligned}
$$

Since the basic scattering involves two photons, one at $\omega_{1}$ and a second at $\omega_{2}$, we need to consider only the second-order term, i.e., $u^{(2)}(t, 0)$ so that

$$
\mathscr{P}_{k}(t)=\left|\left\langle k\left|u^{(2)}(t, 0)\right| n\right\rangle\right|^{2} \text {. }
$$

An alternate method would be to solve for the third-order induced dipole moment of an atom subjected to fields $E_{1}$ exp $\left(i \omega_{1} t\right)$ and $E_{2} \exp \left(i \omega_{2} t\right)$. Specifically, we are looking for an induced dipole moment which is proportional to

$$
E_{1} e^{i \omega_{1} t}\left(E_{1} e^{i \omega t}\right)^{*} E_{2} e^{i \omega_{2} t}=\left|E_{1}\right|^{2} E_{2} e^{i \omega_{2} t} .
$$

Since the expectation value of the projection along the $i$ axis of the dipole moment operator is given by

$$
\left\langle\mu_{i}\right\rangle=\left\langle\psi(t)\left|\mu_{i}\right| \psi(t)\right\rangle .
$$

The desired dipole moment will result from terms such as $\left\langle\psi^{(1)}\left|\mu_{i}\right| \psi^{(2)}\right\rangle,\left\langle\psi^{(0)}\left|\mu_{i}\right| \psi^{(3)}\right\rangle$ where

$$
\psi^{(n)}(t) \equiv u^{(n)}(t, 0) \psi(0)
$$

since they involve the third power of the perturbation $v(t)$.

Using the first approach the power per unit volume generated at $\omega_{2}$ is

$$
p\left(\omega_{2}\right)=N W_{n \rightarrow k} \hbar \omega_{2}
$$

where

$$
W_{n \rightarrow k}=\frac{d}{d t} \mathcal{P}_{k}(t)
$$

is the transition rate per atom from $|n\rangle$ to $|k\rangle$, and $N$ is the density of atoms.

Using the second approach, i.e., the one leading to (11), we calculate the power dissipation at $\omega_{2} p\left(\omega_{2}\right)=E_{2 i} d P_{i}\left(\omega_{2}\right) / d t$, where $P_{i}$ is the polarization at $\omega_{2}$, so that

$$
p\left(\omega_{2}\right)=\frac{1}{2} \overline{E_{2 i} \frac{\partial}{\partial t} N\left\langle\mu_{i}\right\rangle}
$$

where the bar denotes time averaging.

Either result, i.e., (13) or (14), can be obtained in a quick and straightforward manner using Feynman diagrams. This makes it possible to limit the considerations to resonant terms which dominate the process, when such terms exist, while ignoring the remaining terms.

By insisting that the results of (13) and (14) agree with each other we will learn how to include finite linewidths in the analysis.
To calculate the expectation value of physical observables as in (11) we need to obtain an expression for the wavefunction to any desired order of perturbation. The optical field at the atom site is taken as

$$
E(t)=\frac{1}{2} E_{1} e^{i \omega_{1} t}+\frac{1}{2} E_{2} e^{i \omega_{2} t}+\text { c.c. }
$$

and the interaction Hamiltonian as

$$
v(t)=-\bar{\mu} \cdot\left(\frac{\bar{E}_{1}}{2} e^{i \omega_{1} t}+\frac{\bar{E}_{2}}{2} e^{i \omega_{2} t}+\text { c.c. }\right) .
$$

The perturbation is assumed turned on at $t_{0}=-\infty$ at which time the atom is in its ground state $n$. The eigenfunction at a subsequent time $t$ is

$$
\psi(t)=\psi^{(0)}(t)+\psi^{(1)}(t)+\cdots \psi^{(n)}(t)+\cdots
$$

where from (2) and (6)

$$
\psi^{(n)}(t)=u^{(n)}\left(t, t_{0}\right)|n\rangle .
$$

Using (7) we write

$$
\begin{aligned}
\psi^{(0)}(t)= & \exp \left[-i \frac{H_{0}}{h}\left(t-t_{0}\right)\right]|n\rangle=\exp \left[-i \omega_{n}\left(t-t_{0}\right)\right]|n\rangle \\
\psi^{(1)}(t)= & -\frac{i}{h} \int_{t_{0}}^{t} \exp \left[-\frac{i H_{0}}{\hbar}\left(t-t_{1}\right)\right] v\left(t_{1}\right) \\
& \cdot \exp \left[-\frac{i H_{0}}{\hbar}\left(t_{1}-t_{0}\right)\right] d t_{1}|n\rangle
\end{aligned}
$$

which using (4) becomes

$$
\begin{aligned}
\psi^{(1)}(t)= & -\frac{i}{\hbar} \sum_{m} \int_{t_{0}}^{t}|m\rangle\langle m| \exp \left[-i \omega_{m}\left(t-t_{1}\right)\right] V\left(t_{1}\right) \\
& \cdot \exp \left[-i \omega_{n}\left(t_{1}-t_{0}\right)\right]|n\rangle d t_{1} .
\end{aligned}
$$

Since $V\left(t_{1}\right)$ is, according to (16), a sum of four terms, the integrand in (20) is made up of four terms. One such term, for example, resulting from the part of $V(t)$ involving $E_{1}^{*} \exp$ $\left(-i \omega_{1} t\right)$ will yield

$$
\begin{aligned}
\psi^{(1)}(t)= & \frac{i}{2 \hbar} \sum_{m} \int_{t_{0} \rightarrow-\infty}^{t}\left(\mu_{1}\right)_{m n} E_{1}^{*} \exp i\left(\omega_{m n}-\omega_{1}\right) t_{1} \\
& \cdot \exp \left(-i \omega_{m} t \exp \right)\left(i \omega_{n} t_{0}\right) d t_{1}|m\rangle \\
= & \frac{1}{2 \hbar} \sum_{m}\left(\mu_{1}\right)_{m n} E_{1}^{*} \frac{\exp i\left(-\omega_{1}-\omega_{n}\right) t}{\omega_{m n}-\omega_{1}-i \gamma}|m\rangle
\end{aligned}
$$

where $\mu_{1}$ is the projection of $\boldsymbol{\mu}$ along $\boldsymbol{E}_{1}$. In (21) we added the usual convergence factor $\gamma$ by letting $\omega_{m} \rightarrow \omega_{m}-i \gamma$. This causes the integrand at $t_{1}=-\infty$ to be zero. We will show later that if we leave $\gamma$ as in (21) it will account correctly for the finite lifetime or linewidths of the transition. The constant factor $\exp \left(i \omega_{n} t_{0}\right)$ has been left out since it cancels out (through multiplication by its complex conjugate) in the calculation of physical observables. As mentioned above $\psi^{(1)}(t)$ has three more terms each of a form similar to (21) but involving $+\omega_{1},-\omega_{2}$, and $+\omega_{2}$. These arise from using the 
remaining three terms of the Hamiltonian (16) in (20). We thus have

$$
\begin{aligned}
\psi^{(1)}(t)= & \frac{1}{2 \hbar} \sum_{m}\left\{\left(\mu_{1}\right)_{m n} \frac{E_{1}^{*} \exp \left(i\left(-\omega_{1}-\omega_{n}\right) t\right)|m\rangle}{\omega_{m n}-\omega_{1}-i \gamma}\right. \\
& +\left(\mu_{1}\right)_{m n} \frac{E_{1} \exp \left(i\left(\omega_{1}-\omega_{n}\right) t\right)|m\rangle}{\omega_{m n}+\omega_{1}-i \gamma} \\
& +\left(\mu_{2}\right)_{m n} \frac{E_{2}^{*} \exp \left(i\left(-\omega_{2}-\omega_{n}\right) t\right)|m\rangle}{\omega_{m n}-\omega_{2}-i \gamma} \\
& \left.+\left(\mu_{2}\right)_{m n} \frac{E_{2} \exp \left(i\left(\omega_{2}-\omega_{n}\right) t\right)|m\rangle}{\omega_{m n}+\omega_{2}-i \gamma}\right\} .
\end{aligned}
$$

Using (7) we write the second-order wavefunctions $\psi^{(2)}(t)$ as

$$
\begin{aligned}
\psi^{(2)}(t) & =u^{(2)}\left(t, t_{0}\right)|n\rangle=\left(-\frac{i}{h}\right)^{2} \int_{t_{0}}^{t} \int_{t_{0}}^{t_{1}} d t_{1} d t_{2} \\
\cdot & \exp \left[-i H_{h 0}\left(t-t_{1}\right)\right] V\left(t_{1}\right) \exp \left[-i H_{h 0}\left(t_{1}-t_{2}\right)\right] V\left(t_{2}\right) \\
\cdot & \exp \left[-i H_{0}\left(t_{2}-t_{0}\right)\right]|n\rangle=\left(-\frac{i}{\hbar}\right)^{2} \sum_{m} \sum_{s} \int_{t_{0}}^{t} \int_{t_{0}}^{\left.t_{1}\right\rangle t_{2}} \\
\cdot & \exp \left[-i \omega_{s}\left(t-t_{1}\right)\right]|s\rangle\langle s| v\left(t_{1}\right) \\
\cdot & \exp \left[-i \omega_{m}\left(t_{1}-t_{2}\right)\right]|m\rangle \times\langle m| v\left(t_{2}\right) \\
\cdot & \exp \left[-i \omega_{n}\left(t_{2}-t_{0}\right)\right]|n\rangle d t_{2} d t_{1} .
\end{aligned}
$$

Since $V(t)$ contains four frequency terms and appears twice in (23) the full integration in (23) will yield 16 terms. A typical term, as an example involving the use of $-\mu_{1} E_{1}^{*} e^{-i \omega_{1} t_{2}}$ at $t_{2}$ and $-\mu_{2} E_{2}^{*} e^{-i \omega_{2} t_{1}}$ at $t_{1}$ is

$$
\begin{aligned}
\psi_{-\omega_{1},-\omega_{2}}^{(2)}(t) & =\sum_{m} \sum_{s}\left(\frac{1}{\hbar}\right)^{2} \frac{E_{1}^{*} E_{2}^{*}}{4} \\
& \cdot \frac{\left(\mu_{1}\right)_{m n}\left(\mu_{2}\right)_{s m} \exp i\left(-\omega_{n}-\omega_{1}-\omega_{2}\right)}{\left(\omega_{m n}-\omega_{1}-i \gamma\right)\left(\omega_{s n}-\omega_{1}-\omega_{2}-i \gamma\right)}|s\rangle .
\end{aligned}
$$

A convenient way to represent (24) is through the use of a Feynman diagram, as shown in Fig. 2. Time increases from the bottom to the top. Each solid line segment represents an eigenstate. The atom starts at $t_{0}$ in state $n$, scatters at $t_{2}$ into state $m$ by absorbing a photon at $\omega_{1}$. This scattering is accounted for by the factor $\left(\mu_{1}\right)_{m n} E_{1}^{*} e^{-i \omega_{1} t} /\left(\omega_{m n}-\omega_{1}-i \gamma\right)$ in (24). The next "scattering" is at $t_{1}$ and involves an absorption of a photon at $\omega_{2}$. A negative frequency denotes absorption and is represented by an arrow terminating at a vertex, while an arrow starting at a vertex denotes the emission of a photon. A case where the transition from state $n$ to $s$ involves the absorption of a photon at $\omega_{1}$ and the emission of a photon at $\omega_{2}$, as an example, is shown in Fig. 2(b).

The corresponding contribution to $\psi^{(2)}(t)$ can be written by inspection

$$
\begin{aligned}
& \psi_{-\omega_{1}, \omega_{2}}^{(2)}=\sum_{m} \sum_{s}\left(\frac{1}{\hbar}\right)^{2} \frac{E_{1}^{*} E_{2}}{4} \\
& \cdot \frac{\left(\mu_{1}\right)_{m n}\left(\mu_{2}\right)_{s m} \exp i\left(-\omega_{n}-\omega_{1}+\omega_{2}\right) t}{\left(\omega_{m n}-\omega_{1}-i \gamma\right)\left(\omega_{s n}-\omega_{1}+\omega_{2}-i \gamma\right)}|s\rangle .
\end{aligned}
$$

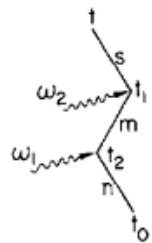

(a)

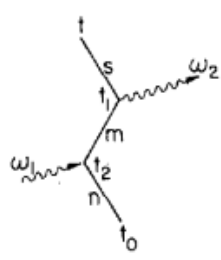

(b)

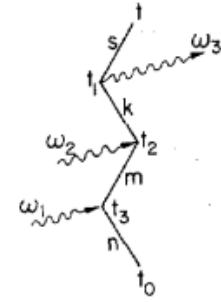

(c)
Fig. 2. The Feynman diagrams used to obtain: (a) $\psi_{-\omega_{1},-\omega_{2}}^{(2)}(t)$. (b) $\psi_{-\omega_{1}}^{(2)},+\omega_{2}(t)$. (c) $\psi_{-\omega_{1},-\omega_{2}, \omega_{3}}^{(3)}(t)$.

We note that each scattering, i.e., each vertex in the diagram contributes one factor to the denominator and the factor is equal to the energy in units of $\hbar$ of the atom and field after the scattering minus the initial $\left(t=t_{0}\right)$ energy. The second factor in the denominator in (25), as an example, is obtained from $\omega_{s}+\omega_{2}-\left(\omega_{1}+\omega_{n}\right)$. The remaining fourteen wavefunctions are obtained by taking all the possible permutations of $\psi_{ \pm \omega_{i}}^{(2)}, \pm \omega_{j}, i, j=1,2$ where our convention is such that $\psi_{+\omega_{i}}^{(2)},-\omega_{j}$, for example, corresponds to emitting an $\omega_{i}$ photon at $t_{2}$ and absorbing an $\omega_{j}$ photon at $t_{1}\left(t_{1}>t_{2}\right)$. We note that $\psi_{-\omega_{j}}^{(2)}, \omega_{i}$ is not equal to $\psi_{\omega_{i},-\omega_{j}}^{(2)}$

Using the diagram technique we can write the wavefunction to any order of perturbation. As an example consider the process in which an atom makes a transition from state $n$ to state $s$ while absorbing one photon at $\omega_{1}$, one photon at $\omega_{2}$, and emitting a photon at $\omega_{3}$. One diagram describing this process is $\psi_{-\omega_{1}}^{(3)},-\omega_{2}, \omega_{3}$, as shown in Fig. 2(c). We obtain by inspection

$$
\begin{aligned}
& \psi_{-\omega_{1},-\omega_{2}, \omega_{3}}^{(3)}=\sum_{m} \sum_{k} \sum_{s} \frac{-E_{1}^{*} E_{2}^{*} E_{3}}{8 \hbar^{3}} \\
& \cdot \frac{\left(\mu_{1}\right)_{m n}\left(\mu_{2}\right)_{k m}\left(\mu_{3}\right)_{s k} \exp i\left(-\omega_{n}-\omega_{1}-\omega_{2}+\omega_{3}\right) t}{\left(\omega_{m n}-\omega_{1}\right)\left(\omega_{k n}-\omega_{1}-\omega_{2}\right)\left(\omega_{s n}-\omega_{1}-\omega_{2}+\omega_{3}\right)}|s\rangle .
\end{aligned}
$$

The total number of $\psi_{ \pm \omega_{i}}^{(3)}, \pm \omega_{j}, \pm \omega_{k}$ combinations is $6^{3}=216$ inside the triple summation. The tremendous advantage of the diagrammatic representation is that it enables us to single out and write down very simply the terms which dominate in any given physical situation. This will become clearer in the examples which follow.

\section{Frequency Addition}

In this section we will calculate the nonlinear susceptibility $d_{i j k}^{\omega_{3}=\omega_{1}+\omega_{2}}$ which describes via the relation

$$
P_{i}^{\omega_{3}=\omega_{1}+\omega_{2}}=d_{i j k}^{\omega_{3}=\omega_{1}+\omega_{2}} E_{2} E_{1}
$$

the induced polarization along the $i$ direction

$$
P_{i}(t)=\operatorname{Re}\left(P_{i}^{\omega_{3}=\omega_{1}+\omega_{2}} e^{i \omega_{3} t}\right)
$$

due to input fields $\operatorname{Re}\left(E_{1 j} e^{i \omega_{1} t}\right)$ and $\operatorname{Re}\left(E_{2 k} e^{i \omega_{2} t}\right)$ polarized along $j$ and $k$, respectively.

The total atomic dipole moment is obtained from

$$
\left\langle\mu_{i}(t)\right\rangle=\left\langle\psi(t)\left|\mu_{i}\right| \psi(t)\right\rangle
$$

so that contributions containing the electric field to second power as in (27) must come from 


$$
\left\langle\mu_{i}^{(2)}(t)\right\rangle=\left\langle\psi^{(0)}\left|\mu_{i}\right| \psi^{(2)}\right\rangle+\left\langle\psi^{(2)}\left|\mu_{i}\right| \psi^{(0)}\right\rangle+\left\langle\psi^{(1)}\left|\mu_{i}\right| \psi^{(1)}\right\rangle
$$

Each term in (30) contributes two terms to the desired polarization-one in which $E_{1}$ scatters first and then $E_{2}$, and vice versa.

Consider as an example the terms due to $\left\langle\psi^{(1)}\left|\mu_{i}\right| \psi^{(1)}\right\rangle$ :

$$
\begin{aligned}
\left\langle\psi^{(1)}\left|\mu_{i}\right| \psi^{(1)}\right\rangle= & \sum_{s} \sum_{m} \frac{1}{4 \hbar^{2}}\langle s| E_{2}\left(\mu_{k}\right)_{s n} \\
& \cdot \frac{\exp i\left(\omega_{2}+\omega_{n}\right) t}{\omega_{s n}-\omega_{2}+i \gamma}\left|\mu_{i}\right| E_{1}\left(\mu_{j}\right)_{m n} \\
& \cdot \frac{\exp i\left(\omega_{1}-\omega_{n}\right) t}{\omega_{m n}+\omega_{1}-i \gamma}|m\rangle=\sum_{s} \sum_{m} \frac{E_{1} E_{2}}{4 \hbar^{2}} \\
& \cdot \frac{\left(\mu_{k}\right)_{s n}\left(\mu_{i}\right)_{s m}\left(\mu_{j}\right)_{m n}}{\left(\omega_{s n}-\omega_{2}+i \gamma\right)\left(\omega_{m n}+\omega_{1}-i \gamma\right)} \\
& \cdot \exp i\left(\omega_{1}+\omega_{2}\right) t .
\end{aligned}
$$

Note that any constant premultiplying a wavefunction to the left of $\mu_{i}$ is replaced by its complex conjugate. Collecting all the terms contributing to $d_{i j k}$ yields

$$
\begin{aligned}
d_{i j k}^{\omega_{3}=\omega_{1}+\omega_{2}=}= & \frac{N}{2 \hbar^{2}} \sum_{m} \sum_{s} \\
& \cdot\left[\frac{\left(\mu_{k}\right)_{s n}\left(\mu_{i}\right)_{s m}\left(\mu_{j}\right)_{m n}}{\left(\omega_{s n}-\omega_{2}+i \gamma\right)\left(\omega_{m n}+\omega_{1}-i \gamma\right)}\right. \\
& +\frac{\left(\mu_{j}\right)_{s n}\left(\mu_{i}\right)_{s m}\left(\mu_{k}\right)_{m n}}{\left(\omega_{s n}-\omega_{1}+i \gamma\right)\left(\omega_{m n}+\omega_{2}-i \gamma\right)} \\
& +\frac{\left(\mu_{j}\right)_{m n}\left(\mu_{k}\right)_{s m}\left(\mu_{i}\right)_{n s}}{\left(\omega_{m n}+\omega_{1}-i \gamma\right)\left(\omega_{s n}+\omega_{1}+\omega_{2}-i \gamma\right)} \\
& +\frac{\left(\mu_{k}\right)_{m n}\left(\mu_{j}\right)_{s m}\left(\mu_{i}\right)_{n s}}{\left(\omega_{m n}+\omega_{2}-i \gamma\right)\left(\omega_{s n}+\omega_{1}+\omega_{2}-i \gamma\right)} \\
& +\frac{\left(\mu_{j}\right)_{n m}\left(\mu_{k}\right)_{s m}\left(\mu_{i}\right)_{n s}}{\left(\omega_{m n}-\omega_{1}+i \gamma\right)\left(\omega_{s n}-\omega_{1}-\omega_{2}+i \gamma\right)} \\
& \left.+\frac{\left(\mu_{k}\right)_{n m}\left(\mu_{j}\right)_{s m}\left(\mu_{i}\right)_{n s}}{\left(\omega_{m n}-\omega_{2}+i \gamma\right)\left(\omega_{s n}-\omega_{1}-\omega_{2}+i \gamma\right)}\right]
\end{aligned}
$$

A more conventional derivation is given in [2] - [4] .

\section{Two-PHoton ABSORPTION}

Here we will apply our formalism to derive the transition rate for absorption of two photons-one at $\omega_{1}$ and a second at $\omega_{2}$-by an atom. By allowing $\omega_{1}=\omega_{2}$ we will get the familiar expression for a two-photon absorption coefficient.

We start by writing the second-order wavefunction $\psi^{(2)}(t)$ corresponding to an atom which at $t=0$ is at state $n$ and which interacts with the radiation field, (15), consisting of fields at $\omega_{1}$ and $\omega_{2}$. We will assume that the largest contribution to $\psi^{(2)}$ comes from $\psi_{-\omega_{1},-\omega}^{(2)}$ corresponding to the diagram of Fig. 3. We write by inspection

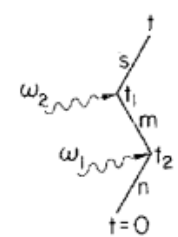

Fig. 3. Feynman diagram used in treating two-photon absorption.

$$
\begin{aligned}
& \psi_{-\omega_{1},-\omega_{2}}^{(2)}(t)=\sum_{m} \sum_{s}\left(\frac{E_{1}^{*} E_{2}^{*}}{4 \hbar^{2}}\right) \\
& \cdot \frac{\left(\mu_{1}\right)_{m n}\left(\mu_{2}\right)_{s m} e^{-i \omega_{s} t}\left[\exp i\left(\omega_{s n}-\omega_{1}-\omega_{2}\right) t-1\right]}{\left(\omega_{m n}-\omega_{1}\right)\left(\omega_{s n}-\omega_{1}-\omega_{2}\right)}|s\rangle .
\end{aligned}
$$

The -1 term is due to the fact that here we integrated from 0 to $t$. Let us assume that for some level $m, \omega_{m n} \simeq \omega_{1}$ so the term $m$ dominates and we rewrite (31) as

$$
\begin{aligned}
& \psi_{-\omega_{1},-\omega_{2}}^{(2)}(t) \simeq \frac{E_{1}^{*} E_{2}^{*}}{4 \hbar^{2}} \sum_{s} \\
& \frac{\left(\mu_{1}\right)_{m n}\left(\mu_{2}\right)_{s m} e^{-i \omega_{s} t} \exp \left[i\left(\omega_{s n}-\omega_{1}-\omega_{2}\right) t-1\right]}{\left(\omega_{m n}-\omega_{1}\right)\left(\omega_{s n}-\omega_{1}-\omega_{2}\right)}|s\rangle .
\end{aligned}
$$

The probability of finding the atom in some state $k$ at time $t$ is $|\langle k \mid \psi(t)\rangle|^{2}$ so that the transition probability due to twophoton absorption is

$$
\begin{aligned}
\mathscr{P}_{k} & =\left|\left\langle k \mid \psi^{(2)}(t)\right\rangle\right|^{2} \\
& =\frac{\left|E_{1}\right|^{2}\left|E_{2}\right|^{2}}{16 h^{4}} \frac{\left(\mu_{1}\right)_{m n}^{2}\left(\mu_{2}\right)_{k m}^{2} \sin ^{2}\left[\frac{1}{2}\left(\omega_{k n}-\omega_{1}-\omega_{2}\right) t\right]}{\left(\omega_{m n}-\omega_{1}\right)^{2}\left[\frac{1}{2}\left(\omega_{k n}-\omega_{1}-\omega_{2}\right)\right]^{2}} .
\end{aligned}
$$

We note that transitions occur to the state $k$ which conserves energy, i.e., to that state where $E_{k}-E_{n}=\not h\left(\omega_{1}+\omega_{2}\right)$. If the normalized lineshape function for the transition $n \rightarrow k$ is $g\left(\omega_{k n}\right)$ then the average value of $P_{k}$ is obtained by multiply. ing (34) by $g\left(\omega_{k n}\right)$ and integrating from $-\infty$ to $\infty$. Using

$$
\lim _{t \rightarrow \infty} \frac{\sin ^{2} \frac{x t}{2}}{(x / 2)^{2}} \longrightarrow 2 \pi t \delta(x)
$$

we obtain for the transition rate

$$
\begin{aligned}
W_{n \rightarrow k} & =\frac{d}{d t} \mathscr{P}_{k} \\
& =\frac{\pi\left|E_{1}\right|^{2}\left|E_{2}\right|^{2}\left(\mu_{1}\right)_{m n}^{2}\left(\mu_{2}\right)_{m k}^{2} g\left(\omega_{k n}=\omega_{1}+\omega_{2}\right)}{8 \hbar^{4}\left(\omega_{m n}-\omega_{1}\right)^{2}} .
\end{aligned}
$$

The special case of one frequency two-photon absorption where $\omega_{1}=\omega_{2}$ has been considered in some detail [5]. We may obtain the absorption coefficient for this case by equat- 
ing the change in the intensity $I_{\omega}$ of $E_{1}$ to the number of transitions per unit time per unit volume.

$$
\frac{d I_{\omega}}{d z}=2 \hbar \omega W_{n \rightarrow k}\left(N_{k}-N_{n}\right)
$$

where $I_{\omega}$ is the intensity $\left(\mathrm{W} / \mathrm{m}^{2}\right)$ at $\omega$, and $N_{k}$ and $N_{n}$ are the population densities of level $k$ and $n$, respectively. Using $I_{\omega}=$ $\left(\epsilon c / n_{1}\right)\left|E_{1}\right|^{2} / 2$ we obtain

$$
\underset{\substack{\text { two } \\ \text { photon }}}{ }=\frac{\pi \omega\left(N_{n}-N_{k}\right)\left(\mu_{1}\right)_{m n}^{2}\left(\mu_{1}\right)_{k m}^{2} n_{1}^{2} g\left(\omega_{k n}=2 \omega\right) I_{\omega}}{\hbar^{3}\left(\omega_{m n}-\omega\right)^{2} \epsilon^{2} c^{2}} .
$$

\section{RAMAN Processes}

As a more detailed example of the diagram formalism we consider the case of Raman processes in which an atom initially in the ground state $n$ absorbs a photon at $\omega_{1}$, emits one at $\omega_{2}$, while making a transition to the state $k$. The situation is depicted in Fig. 4. The situation is identical to that considered in the previous section, except that here the second photon at $\omega_{2}$ is emitted rather than absorbed. This causes the $\omega_{2}$ arrow in the Feynman diagram of Fig. 4 to have the opposite sense to that in Fig. 3. We can use directly the result of Section III and merely reverse the sign of $\omega_{2}$. Using (34) we obtain for the transition probability

$$
\mathscr{P}_{k}(t)=\frac{\left|E_{1}\right|^{2}\left|E_{2}\right|^{2}\left(\mu_{1}\right)_{m n}^{2}\left(\mu_{2}\right)_{k m}^{2}}{16 \hbar^{4}\left(\omega_{m n}-\omega_{1}\right)^{2}} 2 \pi t \delta\left(\omega_{k n}-\omega_{1}+\omega_{2}\right) \text {. }
$$

In (39) it was assumed that one term $m$ dominates, so that the summation over $m$ is omitted. As in (38) we introduce the normalized Raman lineshape function $g\left(\omega_{k n}\right)$ such that $g\left(\omega_{k n}\right) d \omega_{k n}$ is the probability of having $\omega_{k n}$ within the interval $d \omega_{k n}$. Multiplying (39) by $g\left(\omega_{k n}\right) d \omega_{k n}$ and integrating leads to

$$
\rho_{k}(t)=\frac{\pi t}{8 \hbar^{4}} \frac{\left|E_{1}\right|^{2}\left|E_{2}\right|^{2}\left(\mu_{1}\right)_{m n}^{2}\left(\mu_{2}\right)_{k m}^{2}}{\left(\omega_{m n}-\omega_{1}\right)^{2}} g\left(\omega_{k n}=\omega_{1}-\omega_{2}\right)
$$

and a corresponding transition rate

$$
\begin{aligned}
W_{n \rightarrow k}= & \frac{d \Phi_{k}}{d t}=\frac{\pi}{8 \hbar^{4}} \frac{\left|E_{1}\right|^{2}\left|E_{2}\right|^{2}\left(\mu_{1}\right)_{m n}^{2}\left(\mu_{2}\right)_{k m}^{2}}{\left(\omega_{m n}-\omega_{1}\right)^{2}} \\
& \cdot g\left(\omega_{k n}=\omega_{1}-\omega_{2}\right) \\
= & \frac{\pi n_{1} n_{2} I_{1} I_{2}\left(\mu_{1}\right)_{m n}^{2}\left(\mu_{2}\right)_{k m}^{2}}{2 \hbar^{4} c^{2} \epsilon_{1} \epsilon_{2}\left(\omega_{m n}-\omega_{1}\right)^{2}} g\left(\omega_{k n}=\omega_{1}-\omega_{2}\right)
\end{aligned}
$$

where $I$ is the intensity and $\epsilon_{1}$ and $\epsilon_{2}$ the dielectric constants at $\omega_{1}$ and $\omega_{2}$, respectively, and $n_{1}$ and $n_{2}$ are the refractive indices.

The exponential gain coefficient describing the amplification of the field at $\omega_{2}$ is obtained from

$$
\frac{d I_{2}}{d z}=g_{R} I_{2}=W_{n \rightarrow k}\left(N_{n}-N_{k}\right) \hbar \omega_{2}
$$
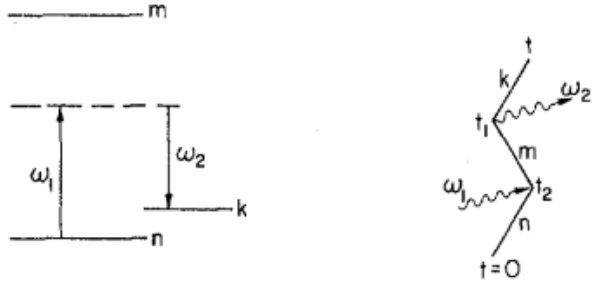

Fig. 4. Energy levels and Feynman diagrams involved in Raman scattering.

which, using (42) gives

$$
\begin{aligned}
g_{R}= & \frac{\pi n_{1} n_{2} \omega_{2}\left(\mu_{1}\right)_{m n}^{2}\left(\mu_{2}\right)_{k m}^{2}\left(N_{n}-N_{k}\right) I_{1}}{2 \hbar^{3}} \frac{c^{2} \epsilon_{1} \epsilon_{2}\left(\omega_{m n}-\omega_{1}\right)^{2}}{} \\
& g\left(\omega_{k n}=\omega_{1}+\omega_{2}\right) .
\end{aligned}
$$

Next we will describe the same Raman scattering leading to (43) by a different approach. Instead of using the concept of a transition rate we will calcualte the induced dipole moment at $\omega_{2}$ of an atom interacting with the $\omega_{1}$ and $\omega_{2}$ fields. To make sure that we treat the problem to the same order of interaction we look for a dipole moment cubic in the applied field amplitudes.

$$
\begin{aligned}
\left\langle\mu_{i}\right\rangle & \propto E_{1} E_{1}^{*} E_{2} \exp i\left(\omega_{1}-\omega_{1}+\omega_{2}\right) t \\
& =\left|E_{1}\right|^{2} E_{2} e^{i \omega_{2} t} .
\end{aligned}
$$

Such a contribution to $\mu_{i}$ which involves the third power of $E$ can come from combinations such as $\left\langle\psi^{(1)}\left|\mu_{i}\right| \psi^{(2)}\right\rangle$, $\left\langle\psi^{(0)}\left|\mu_{i}\right| \psi^{(3)}\right\rangle$. An examination of (22) and (25) shows that the largest dipole moment will arise from the use of

$$
\begin{aligned}
\left\langle\mu_{i}\right\rangle=\left\langle\psi^{(1)}(t)\left|\mu_{i}\right| \psi^{(2)}\right\rangle+\text { c.c. } & \\
\psi^{(1)}(t)= & \sum_{m}\left(\frac{1}{2 \hbar}\right) \frac{E_{1}^{*}\left(\mu_{1}\right)_{m n}}{\left(\omega_{m n}-i \gamma-\omega_{1}\right)} \exp i\left(-\omega_{1}-\omega_{n}\right) t|m\rangle \\
\psi^{(2)}(t)= & \sum_{g} \sum_{s} \frac{E_{1}^{*} E_{2}}{4 \hbar^{2}} \\
& \cdot \frac{\left(\mu_{1}\right)_{g n}\left(\mu_{2}\right)_{s g} \exp i\left(-\omega_{1}+\omega_{2}-\omega_{n}\right) t}{\left(\omega_{g n}-\omega_{1}-i \gamma\right)\left(\omega_{s n}-\omega_{1}+\omega_{2}-i \gamma\right)}|s\rangle
\end{aligned}
$$

so that using (44)

$$
\begin{aligned}
& \left\langle\mu_{i}\right\rangle=\frac{1}{8 \hbar^{3}} \sum_{m} \sum_{g} \sum_{s} E_{1} E_{1}^{*} E_{2} \\
& \cdot \frac{\left(\mu_{1}\right)_{m n}\left(\mu_{i}\right)_{m s}\left(\mu_{1}\right)_{g n}\left(\mu_{2}\right)_{s g}}{\left(\omega_{m n}-\omega_{1}\right)\left(\omega_{g n}-\omega_{1}\right)\left(\omega_{s n}-\omega_{1}+\omega_{2}-i \gamma\right)} e^{i \omega_{2} t}+\text { c.c. }
\end{aligned}
$$

where we dropped the term $i \gamma$ in the first two factors in the denominators. These must be reintroduced if operation is such that $\omega_{m n} \cong \omega_{1}$ or $\omega_{g n} \cong \omega_{1}$.

We will assume that the summation over $m$ is dominated by one term where $\omega_{m n} \simeq \omega_{1}$. Also, that $\omega_{1}$ and $\omega_{2}$ are chosen so that for some level $s=k \omega_{k n}=\omega_{1}-\omega_{2}$. We thus simplify (47) to 


$$
\begin{aligned}
\left\langle\mu_{i}\right\rangle= & \frac{1}{8 \hbar^{3}} \frac{\left(\mu_{1}\right)_{m n}^{2}\left(\mu_{i}\right)_{m k}\left(\mu_{2}\right)_{k m} e^{i \omega_{2} t}}{\left(\omega_{m n}-\omega_{1}\right)^{2}\left(\omega_{k n}-\omega_{1}+\omega_{2}-i \gamma\right)} \\
& \cdot E_{1} E_{1}^{*} E_{2}+\text { c.c. }
\end{aligned}
$$

We define a nonlinear Raman susceptibility by

$$
P_{i}^{\left(\omega_{2}\right)}=N\left\langle\mu_{i}\right\rangle=\epsilon_{0} \chi_{R}\left|E_{1}\right|^{2} E_{2}
$$

where $P^{\left(\omega_{2}\right)}$ is the complex amplitude of the polarization at $\omega_{2}$. From (48) and (49) we obtain

$$
\chi_{R}=\frac{N}{4 \hbar^{3} \epsilon_{0}} \frac{\left(\mu_{1}\right)_{m n}^{2}\left(\mu_{i}\right)_{m k}\left(\mu_{2}\right)_{m k}}{\left(\omega_{m n}-\omega_{1}\right)^{2}\left(\omega_{k n}-\omega_{1}+\omega_{2}-i \gamma\right)} .
$$

The average power per unit volume generated at $\omega_{2}$ is

$$
p=-\frac{\omega_{2} \epsilon_{0}}{2}\left|E_{2}\right|^{2}\left|E_{1}\right|^{2} \chi_{R}^{\prime \prime}
$$

where

$$
\chi_{R}=\chi_{R}^{\prime}-i \chi_{R}^{\prime \prime} \text {. }
$$

An examination of (50) shows that when $\gamma=0, \chi_{R}$ is real and $p=0$. The convergence factor $\gamma$ in the Feynman formalism is thus fundamentally related to the problem of power dissipation. Let us for a moment consider $\gamma$ as a parameter and calculate the power $p$. We obtain

$$
\begin{aligned}
& \chi_{R}^{\prime}=\frac{N}{4 \hbar^{3} \epsilon_{0}} \frac{\left(\mu_{1}\right)_{m n}^{2}\left(\mu_{i}\right)_{m k}\left(\mu_{2}\right)_{m k}\left[\omega_{k n}-\left(\omega_{1}-\omega_{2}\right)\right]}{\left(\omega_{m n}-\omega_{1}\right)^{2}\left[\left(\omega_{k n}-\omega_{1}+\omega_{2}\right)^{2}+\gamma^{2}\right]} \\
& \chi_{R}^{\prime \prime}=\frac{-N}{4 \hbar^{3} \epsilon_{0}} \frac{\left(\mu_{1}\right)_{m n}^{2}\left(\mu_{i}\right)_{m k}\left(\mu_{2}\right)_{m k} \gamma}{\left(\omega_{m n}-\omega_{1}\right)^{2}\left[\left(\omega_{k n}-\omega_{1}+\omega_{2}\right)^{2}+\gamma^{2}\right]}
\end{aligned}
$$

and from (51) (putting $i=2$ since only the polarization component along $E_{2}$ contributes to absorption)

$$
p=\frac{\omega_{2}}{8 \hbar^{3}}\left|E_{1}\right|^{2}\left|E_{2}\right|^{2} \frac{N\left(\mu_{1}\right)_{m n}^{2}\left(\mu_{2}\right)_{m k}^{2} \gamma}{\left(\omega_{m n}-\omega_{1}\right)^{2}\left[\left(\omega_{k n}-\omega_{1}+\omega_{2}\right)^{2}+\gamma^{2}\right]} .
$$

This power must be the same as that calculated using the transition rate approach leading to (41). The latter leads to

$$
\begin{aligned}
p & =N W_{n \rightarrow k} \hbar \omega_{2} \\
& =\frac{N \pi \omega_{2}}{8 \hbar^{3}} \frac{\left|E_{1}\right|^{2}\left|E_{2}\right|^{2}\left(\mu_{1}\right)_{m n}^{2}\left(\mu_{2}\right)_{k m}^{2}}{\left(\omega_{m n}-\omega_{1}\right)^{2}} g\left(\omega_{k n}=\omega_{1}-\omega_{2}\right) .
\end{aligned}
$$

Equating (54) to (55) and taking $\mu_{i}=\mu_{2}$ gives

$$
\frac{\pi / \gamma}{\left[\omega_{k n}-\left(\omega_{1}-\omega_{2}\right)\right]^{2}+\gamma^{2}}=g\left[\omega_{k n}-\left(\omega_{1}-\omega_{2}\right)\right] .
$$

We thus obtained an explicit expression for the transition lineshape function $g\left[\omega_{k n}-\left(\omega_{1}-\omega_{2}\right)\right]$. We now find that the result of the coherent treatment in which the problem is treated by the induced dipole agrees exactly with the transition rate approach if we merely regard $\gamma$ as the Lorentzian width of the $k \rightarrow n$ transition.

The correct form of the lineshape function $\chi_{R}^{\prime \prime}$ and its sign depend on the sign of $\gamma$ in (53). We find that the procedure followed yielded the correct result. In this procedure we chose the sign of $\gamma$ to insure that $\psi(t)$ does not depend on the "starting" time $t_{0} \rightarrow-\infty$ and then simply limited the derivation (a)

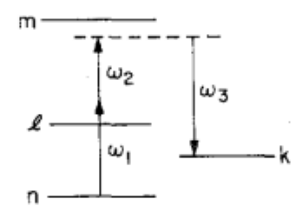

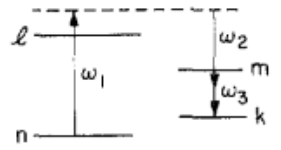

(b)
Fig. 5. Energy levels and Feynman diagrams involved in two-photon Raman scattering.

to terms for which $\omega_{k n} \simeq \omega_{1}-\omega_{2}$, i.e., total (photon + atom) energy conservation. This last consideration automatically results in the use of $\psi^{(2)}$ in (44) as a ket vector.

If we apply the same procedure to derive the nonlinear susceptibility at the anti-Stokes frequency $\omega_{3}=\omega_{1}+\omega_{k n}$ ( $\omega_{1}=$ laser frequency, $k$ and $n$ are the levels shown in Fig. 4) we obtain

$$
\left\langle\mu_{i}^{\left(\omega_{3}\right)}\right\rangle=\left\langle\psi_{-\omega_{3}, \omega_{1}}^{(2)}\left|\mu_{i}\right| \psi_{\omega_{!}}^{(1)}\right\rangle
$$

as the only term oscillating at $\omega_{3}$ with an energy conserving denominator $\left(\omega_{k n}-\omega_{3}+\omega_{1}\right)$.

We define a nonlinear anti-Stokes susceptibility $\chi_{A S}$ by

$$
P_{i}^{\left(\omega_{3}\right)}=N\left\langle\mu_{i}^{\left(\omega_{3}\right)}\right\rangle=\epsilon_{0} \chi_{A S}\left|E_{1}\right|^{2} E_{3}
$$

and obtain by a procedure similar to that leading to (48) and (50)

$$
\begin{aligned}
\chi_{A S}= & \frac{N}{4 h^{3} \epsilon_{0}} \\
& \cdot\left[\sum_{m} \sum_{g} \frac{\left(\mu_{i}\right)_{k m}\left(\mu_{3}\right)_{g m}\left(\mu_{1}\right)_{g k}\left(\mu_{1}\right)_{m n}}{\left(\omega_{g n}-\omega_{3}+i \gamma_{g n}\right)\left(\omega_{m n}+\omega_{1}-i \gamma_{m n}\right)}\right] \\
& \cdot\left(\frac{1}{\omega_{k n}-\omega_{3}+\omega_{1}+i \gamma}\right) .
\end{aligned}
$$

We note that instead of having $-i \gamma$ in the last factor as in (50) we now have $+i \gamma$. This is due to the use of $\psi_{-\omega_{3}}^{(2)}, \omega_{1}$ as a bra rather than a ket. It follows directly that the sign of $\chi_{A S}^{\prime \prime}$ is opposite to that of $\chi_{R}^{\prime \prime}$ and, consequently, that anti-Stokes radiation at $\omega_{3}$ in the presence of a laser field is attenuated.

We are thus encouraged to believe, on the basis of the Raman examples, that the procedure described above leads to the correct lineshape functions. Any lingering doubt can always be resolved by comparing the result against those obtained by the use of the density matrix formalism [7] .

\section{Two-Photon Raman Processes}

As the last application of the evolution operator techniques we consider the problem of two-photon Raman transitions of the type considered in Fig. 5. These processes were described by Yatsiv et al. [6]. We shall expand this discussion and show how these processes can be described using the formalism of nonlinear optics.

In the first of these processes [Fig. 5(a)] one photon at $\omega_{1}$ and one photon at $\omega_{2}$ are absorbed and a photon at $\omega_{3}$ is emitted, while the atom (molecule) makes a transition from the initial state $n$ to some final state $k$. In the second process one photon $\left(\omega_{1}\right)$ is absorbed while photons $\omega_{2}$ and $\omega_{3}$ are emitted during the same transition. 
The analysis of both these processes is similar and the results for Fig. 5(b) can be obtained from those of Fig. 5(a) by reversing the sign of $\omega_{2}$.

Using the formalism of nonlinear optics, the scattering events of Fig. 5 can be represented by

$$
\begin{aligned}
p^{\omega_{3}=\omega_{1}-\omega_{1}+\omega_{2}-\omega_{2}+\omega_{3}=} & \epsilon_{0} \chi_{R 2}^{\left(\omega_{3}=\omega_{1}-\omega_{1}+\omega_{2}-\omega_{2}+\omega_{3}\right)} \\
& \cdot\left|E_{1}\right|^{2}\left|E_{2}\right|^{2} E_{3}
\end{aligned}
$$

so that we may expect an amplification (or attenuation) at $\omega_{3}$ with an exponential constant proportional to $\left|E_{1}\right|^{2}\left|E_{2}\right|^{2}$ in analogy with the one-photon Raman process (43). Alternatively, we can, as in Section IV, obtain the gain or loss by calculating the transition rate per atom for the process illustrated in Fig. 5(a).

By direct analogy with the calculation leading to (39) we obtain for the probability $P_{i c}(t)$ of finding an atom in state $k$

$$
\begin{aligned}
\mathscr{P}_{k}= & \left|\left\langle k \mid \psi^{(3)}(t)\right\rangle\right|^{2}=\frac{\left|E_{1}\right|^{2}\left|E_{2}\right|^{2}\left|E_{3}\right|^{2}\left(\mu_{1}\right)_{m n}^{2}\left(\mu_{2}\right)_{m s}^{2}\left(\mu_{3}\right)_{s k}^{2}}{2^{6} \hbar^{6}\left(\omega_{m n}-\omega_{1}\right)^{2}\left(\omega_{s n}-\omega_{1}-\omega_{2}\right)^{2}} \\
& \cdot 2 \pi t \delta\left(\omega_{k n}-\omega_{1}-\omega_{2}+\omega_{3}\right)
\end{aligned}
$$

so that the transition rate $W_{n \rightarrow k}$ is

$$
\begin{aligned}
W_{n \rightarrow k}= & \frac{\pi I_{1} I_{2} I_{3}\left(\mu_{1}\right)_{m n}^{2}\left(\mu_{2}\right)_{m s}^{2}\left(\mu_{3}\right)_{s k}^{2} n_{1} n_{2} n_{3}}{4 \hbar^{6} c^{3} \epsilon^{3}\left(\omega_{m n}-\omega_{1}\right)^{2}\left(\omega_{s n}-\omega_{1}-\omega_{2}\right)^{2}} \\
& \cdot g\left(\omega_{k n}=\omega_{1}+\omega_{2}-\omega_{3}\right) .
\end{aligned}
$$

The power per unit volume generated at $\omega_{3}$ is thus

where the two-photon Raman gain $g_{R^{2}}$ is

$$
p\left(\omega_{3}\right)=W_{n \rightarrow k} N_{n} \hbar \omega_{3}=g_{R 2} I_{3}
$$

$$
g_{R 2}=g_{R} \frac{\mu^{2} I_{2} n_{3}}{2 h^{2} c \epsilon\left(\omega_{s n}-\omega_{1}-\omega_{2}\right)^{2}} .
$$

If we assume that $I_{2} \sim 10^{6} \mathrm{~W} / \mathrm{cm},\left(\omega_{s n}-\omega_{1}-\omega_{3}\right) \sim 10^{10}$, i.e., near-resonant tuning, $\mu \sim 10^{-30} \mathrm{MKS}$, we obtain

$$
g_{R 2}=g_{R}
$$

so that, usin intense laser sources tuned near atomic resonances, it may be possible to obtain two-photon Raman gain approaching those of normal Raman processes.

Unlike frequency addition and multiplication, this process does not require phase matching. This is due to the fact that if the spatial phase factors are included in (57) they will cancel out, since each field amplitude is multiplied by its complex conjugate except for $E_{3}$. The induced polarization $P^{\left(\omega_{3}\right)}$ thus has the same spatial phase as $E_{3}$ and the interaction is spatially cumulative. If the interaction region is provided with feedback, oscillation at $\omega_{3}$ may result.

To describe the same process using the concept of nonlinear Raman susceptibility, we proceed to derive the dipole moment per atom $\left\langle\mu_{i}\right\rangle$ at $\omega_{3}$ induced in the presence of fields at $\omega_{1}$, $\omega_{2}$, and $\omega_{3} \cdot$ Resonant contributions to the fifth-order dipole moment arise from

$$
\left\langle\mu_{i}\right\rangle=\left\langle\psi_{-\omega_{1},-\omega_{2}}^{(2)}\left|\mu_{i}\right| \psi_{-\omega_{1},-\omega_{2}, \omega_{3}}^{(3)}\right\rangle+\text { c.c. }
$$

From (25) and (26) we obtain

$$
\begin{aligned}
\psi_{-\omega_{1},-\omega_{2}}^{(2)}(t)= & \sum_{g} \sum_{s}\left(\frac{1}{2 \hbar}\right)^{2} \\
& \frac{E_{1}^{*} E_{2}^{*} \exp i\left(-\omega_{1}-\omega_{2}-\omega_{n}\right) t\left(\mu_{1}\right)_{n g}\left(\mu_{2}\right)_{g s}}{\left(\omega_{g n}-\omega_{1}-i \gamma\right)\left(\omega_{s n}-\omega_{1}-\omega_{2}-i \gamma\right)}|s\rangle
\end{aligned}
$$

so that

$$
\psi_{-\omega_{1},-\omega_{2}, \omega_{3}}^{(3)}(t)=\frac{1}{8 \hbar^{3}} \sum_{l} \sum_{m} \sum_{k} \frac{E_{1}^{*} E_{2}^{*} E_{3} \exp i\left(-\omega_{1}-\omega_{2}+\omega_{3}-\omega_{n}\right) t\left(\mu_{1}\right)_{n l}\left(\mu_{2}\right)_{l m}\left(\mu_{3}\right)_{m k}}{\left(\omega_{l n}-\omega_{1}-i \gamma\right)\left(\omega_{m n}-\omega_{1}-\omega_{2}-i \gamma\right)\left(\omega_{k n}-\omega_{1}-\omega_{2}+\omega_{3}-i \gamma\right)}|k\rangle
$$

$$
\begin{aligned}
\left\langle\mu_{i}\right\rangle & =\left(\frac{1}{32 \hbar^{5}}\right) \sum_{g s i m k} \\
& \times \frac{\left|E_{1}\right|^{2}\left|E_{2}\right|^{2} E_{3}\left(\mu_{1}\right)_{g n}\left(\mu_{2}\right)_{s g}\left(\mu_{1}\right)_{n l}\left(\mu_{2}\right)_{l m}\left(\mu_{3}\right)_{m k}\left(\mu_{i}\right)_{s p} e^{i \omega_{3} t}}{\left(\omega_{l n}-\omega_{1}-i \gamma\right)\left(\omega_{m n}-\omega_{1}-\omega_{2}-i \gamma\right)\left(\omega_{k n}-\omega_{1}-\omega_{2}+\omega_{3}-i \gamma\right)\left(\omega_{g n}-\omega_{1}+i \gamma\right)\left(\omega_{s n}-\omega_{1}-\omega_{2}+i \gamma\right)}+\text { c.c. }
\end{aligned}
$$

$$
\begin{aligned}
g_{R 2}= & \frac{N_{n} \pi \omega_{3}\left(\mu_{1}\right)_{m n}^{2}\left(\mu_{2}\right)_{m s}^{2}\left(\mu_{3}\right)_{s k}^{2} n_{1} n_{2} n_{3} I_{1} I_{2}}{4 h^{5} c^{3} \epsilon^{3}\left(\omega_{m n}-\omega_{1}\right)^{2}\left(\omega_{s n}-\omega_{1}-\omega_{2}\right)^{2}} \\
& \cdot g\left(\omega_{k n}=\omega_{1}+\omega_{2}-\omega_{3}\right)
\end{aligned}
$$

Let us assume that $\omega_{1}$ and $\omega_{2}$ are adjusted so that for some levels $l$ and $m, \omega_{l n} \simeq \omega_{1}$ while $\omega_{m n} \simeq \omega_{1}+\omega_{2}$. Also, that a level $k$ exists such that $\omega_{k n} \simeq \omega_{1}+\omega_{2}-\omega_{3}$. Keeping only the resonant term of (65) we obtain

$$
\left\langle\mu_{i}\right\rangle \simeq \frac{1}{32 \hbar^{5}} \frac{\left|E_{1}\right|^{2}\left|E_{2}\right|^{2} E_{3}\left(\mu_{1}\right)_{l n}^{2}\left(\mu_{2}\right)_{l m}^{2}\left(\mu_{3}\right)_{m k}\left[\omega_{k n}-\left(\omega_{1}+\omega_{2}-\omega_{3}-i \gamma_{k n}\right] e^{i \omega_{3} t}\right.}{\left.\left[\omega_{1}\right)^{2}+\gamma_{l n}^{2}\right]\left[\left(\omega_{m n}-\omega_{1}-\omega_{2}\right)^{2}+\gamma_{m n}^{2}\right]\left[\left\{\omega_{k n}-\left(\omega_{1}+\omega_{2}-\omega_{3}\right)\right\}^{2}+\gamma_{k n}^{2}\right]}+\text { c.c. }
$$

and is thus proportional to the product of intensities at $\omega_{1}$ and $\omega_{2}$.

We note by comparison to the expression (43) for the onephoton (i.e., conventional) Raman gain $g_{R}$ that
If we relate the complex amplitude $P_{i}\left(\omega_{3}\right)$ of the induced polarization $N\left\langle\mu_{i}\right\rangle$ to the complex field amplitudes via

$$
P_{i}^{\left(\omega_{3}\right)}=\epsilon_{0} \chi_{R 2}\left|E_{1}\right|^{2}\left|E_{2}\right|^{2} E_{3}, \quad \chi_{R 2}=\chi_{R 2}^{\prime}-i \chi_{R 2}^{\prime \prime}
$$

we obtain

$$
\begin{aligned}
\chi_{R 2}^{\prime} & =\frac{N}{16 \hbar^{5} \epsilon_{0}} \frac{\left(\mu_{1}\right)_{l n}^{2}\left(\mu_{2}\right)_{l m}^{2}\left(\mu_{3}\right)_{m k}\left(\mu_{i}\right)_{m k}}{\left[\left(\omega_{l n}-\omega_{1}\right)^{2}+\gamma_{l n}^{2}\right]\left[\left(\omega_{m n}-\omega_{1}-\omega_{2}\right)^{2}+\gamma_{m n}^{2}\right]} \frac{\left[\omega_{k n}-\left(\omega_{1}+\omega_{2}-\omega_{3}\right)\right]}{\left[\left\{\omega_{k n}-\left(\omega_{1}+\omega_{2}-\omega_{3}\right)\right\}^{2}+\gamma_{k n}^{2}\right]} \\
\chi_{R 2}^{\prime \prime} & =-\frac{N}{16 \hbar^{5} \epsilon_{0}} \frac{\left(\mu_{1}\right)_{l n}^{2}\left(\mu_{2}\right)_{l m}^{2}\left(\mu_{3}\right)_{m k}\left(\mu_{i}\right)_{m k} \gamma_{k n}}{\left[\left(\omega_{l n}-\omega_{1}\right)^{2}+\gamma_{l n}^{2}\right]\left[\left(\omega_{m n}-\omega_{1}-\omega_{2}\right)^{2}+\gamma_{m n}^{2}\right]\left[\left\{\omega_{k n}-\left(\omega_{1}+\omega_{2}-\omega_{3}\right)\right\}^{2}+\gamma_{k n}^{2}\right]}
\end{aligned}
$$


where $2 \gamma_{i j}$ is the full width at half maximum of the $i \rightarrow j$ transition.

It follows immediately that the presence of a negative $\chi_{R 2}^{\prime \prime}$ causes an amplification at $\omega_{3}$ with an exponential gain constant [7]

$$
\begin{aligned}
g_{R 2}=-\frac{\omega_{3}}{c n_{3}} \chi_{R 2}^{\prime \prime}\left|E_{1}\right|^{2}\left|E_{2}\right|^{2} & \text { (69) } \begin{array}{l}
\text { process of Fig. 5(b) can be used to gene } \\
\text { radiation at } \omega_{3} .
\end{array} \\
& =\frac{N \pi \omega_{3}\left(\mu_{1}\right)_{l n}^{2}\left(\mu_{2}\right)_{l m}^{2}\left(\mu_{3}\right)_{m k}^{2} n_{1} n_{2} n_{3} I_{1} I_{2}}{4 \hbar^{5} c^{3} \epsilon_{1} \epsilon_{2} \epsilon_{3}\left[\left(\omega_{l n}-\omega_{1}\right)^{2}+\gamma_{l n}^{2}\right]\left[\left(\omega_{m n}-\omega_{1}-\omega_{2}\right)^{2}+\gamma_{m n}^{2}\right]} \frac{\gamma_{k n} / \pi}{\left[\left\{\omega_{k n}-\left(\omega_{1}+\omega_{2}-\omega_{3}\right)\right\}^{2}+\gamma_{k n}^{2}\right]}
\end{aligned}
$$

where we used $I_{i}=c \epsilon_{i}\left|E_{i}\right|^{2} / 2 n_{i}$. The result (70) for $g_{R 2}$ is identical with (61) once we associate the second factor in (70) with the normalized lineshape function $g\left[\omega_{k n}-\left(\omega_{1}+\right.\right.$ $\left.\left.\omega_{2}-\omega_{3}\right)\right]$.

We thus find that the gain exercised by the wave $E_{3}$ is proportional to the product $I_{1} I_{2}$ of the intensities at $\omega_{1}$ and $\omega_{2}$, so that a stimulated Raman emission at $\omega_{3}$ may be expected at some critical value of $I_{1} I_{2}$.

The analysis of the two-photon (emissive) Raman process shown in Fig. 5(b) is similar. All we need to do to obtain the gain at $\omega_{3}$ is to replace $\omega_{2}$ by $-\omega_{2}$. A fundamental difference between the two processes, however, would be revealed had we considered the consequence of the quantized nature of the field operators. The temporal rate of the photon number at $\omega_{3}$ corresponding to the process in Fig. 5(a) is

while that of Fig. 5(b) is

$$
\frac{d n_{3}}{d t}=\frac{d n_{2}}{d z}=C_{2} n_{1}\left(n_{2}+1\right)\left(n_{3}+1\right)
$$

where $n_{i}$ is the number of photons at $\omega_{i}, C_{1}$ and $C_{2}$ are rate

$$
\frac{d n_{3}}{d t}=C_{1} n_{1} n_{2}\left(n_{3}+1\right)
$$
sion of photons in Fig. 5(a) requires the simultaneous presence of the fields at $\omega_{1}$ and $\omega_{2}$ while in Fig. $5(\mathrm{~b})$ stimulation can take place with a single input at $\omega_{1}$, since $d n_{3} / d z>0$ when $n_{3}=n_{2}=0$.

We may also note that the emissive two-photon Raman process of Fig. 5(b) can be used to generate long wavelength constants. It follows immediately that the stimulated emis-

To summarize, the formalism of time evolution operators and the related technique of Feynman diagrams was applied to a variety of multiphoton processes in nonlinear optics. The formalism affords a direct and orderly treatment of complicated high-order processes and makes it possible to obtain directly the nonlinear susceptibilities describing these interactions.

\section{REFERENCES}

[1] A. Messiah, Quantum Mechanics II. New York: Interscience, 1962, p. 724.

[2] J. A. Armstrong, N. Bloembergen, J. Ducuing, and P. S. Pershan, "Interaction between light waves in a nonlinear dielectric," Phys. Rev., vol. 127, p. 1918, 1962.

[3] R. B. Miles and S. E. Harris, "Optical third-harmonic generation in alkali metal vapors," IEEE J. Quantum Electron., vol. QE-9, pp. 470-484, Apr. 1973.

[4] R. L. Abrams, A. Yariv, and P. A. Yeh, "Stark induced threewave mixing in molecular gases-Part I: Theory," IEEE J. Quantum Electron., vol. QE-13, pp. 79-82, Mar. 1977.

[5] W. Kaiser and C. G. B. Garrett, Phys. Rev., vol. 7, p. 229, 1961.

[6] S. Yatsiv, M. Rokni, and S. Barak, "S-8 Enhanced and stimulated multiphoton processes in free atoms," IEEE J. Quantum Electron., vol. QE-4, pp. 900-904, Nov. 1968.

[7] A. Yariv, Quantum Electronics, 2nd ed. New York: Wiley, 1975, p. 487.

\title{
Temperature and Wavelength Dependent Properties of Multiplate Reflectors
}

\author{
JOHN B. SHAW AND GAIL A. MASSEY, SENIOR MEMBER, IEEE
}

\begin{abstract}
This paper describes a new approach to the design of multielement resonant reflectors. These mirrors provide an effective means of controlling the spectral spread of high-gain laser oscillators, but the complexity of the reflectivity as a function of wavelength $R(\lambda)$ has made the design of these systems rather difficult. However, we show
\end{abstract}

Manuscript received April 25, 1977; revised August 8, 1977. This work was supported by the National Science Foundation under Grant ENG 73-03795.

The authors are with the Department of Applied Physics and Electronic Science, Oregon Graduate Center, Beaverton, OR 97005. that by making a two-dimensional plot of reflectivity versus wavelength and temperature, one can obtain several parameters of the system immediately that an $R(\lambda)$ plot does not reveal. Simple algebraic expressions for some of these parameters are given, and a configuration for an improved four-element wavelength selector based on these results is presented.

\section{INTRODUCTION}

$\mathbf{M}$ ULTIPLATE resonant reflectors have proved useful as cavity mirrors in high-gain laser oscillators which 